\title{
Evolution of Charge and Pair Density Modulations in Overdoped $\mathrm{Bi}_{2} \mathrm{Sr}_{2} \mathrm{CuO}_{6+\delta}$
}

\author{
Xintong Li, ${ }^{1}$ Changwei Zou, ${ }^{1}$ Ying Ding, ${ }^{2}$ Hongtao Yan, ${ }^{2}$ Shusen Ye, ${ }^{1}$ Haiwei Li, ${ }^{1}$ Zhenqi Hao, \\ Lin Zhao, ${ }^{2}$ Xingjiang Zhou, ${ }^{2}$ and Yayu Wang ${ }^{1,3, *}$ \\ ${ }^{1}$ State Key Laboratory of Low Dimensional Quantum Physics, Department of Physics, \\ Tsinghua University, Beijing 100084, China \\ ${ }^{2}$ Beijing National Laboratory for Condensed Matter Physics, Institute of Physics, \\ Chinese Academy of Sciences, Beijing 100190, People's Republic of China \\ ${ }^{3}$ Frontier Science Center for Quantum Information, Beijing 100084, People's Republic of China
}

(Received 20 February 2020; revised 24 October 2020; accepted 17 November 2020; published 12 January 2021)

\begin{abstract}
One of the central issues concerning the mechanism of high-temperature superconductivity in cuprates is the nature of the ubiquitous charge order and its implications to superconductivity. Here, we use scanning tunneling microscopy to investigate the evolution of charge order from the optimally doped to strongly overdoped $\mathrm{Bi}_{2} \mathrm{Sr}_{2} \mathrm{CuO}_{6+\delta}$ cuprates. We find that, with increasing hole concentration, the long-range checkerboard order gradually evolves into short-range glassy patterns consisting of diluted charge puddles. Each charge puddle has a unidirectional nematic internal structure and exhibits clear pair density modulations as revealed by the spatial variations of the superconducting coherence peak and gap depth. Both the charge puddles and the nematicity vanish completely in the strongly overdoped nonsuperconducting regime, when another type of short-range order with $\sqrt{2} \times \sqrt{2}$ periodicity emerges. These results shed important new light on the intricate interplay between the intertwined orders and the superconducting phase of cuprates.
\end{abstract}

DOI: 10.1103/PhysRevX.11.011007

Subject Areas: Condensed Matter Physics, Strongly Correlated Materials, Superconductivity

\section{INTRODUCTION}

A key characteristic of the cuprate high-temperature superconductors is the strong tendency for the charge carriers to form symmetry-breaking ordered states [1]. In particular, the charge order phenomena are found ubiquitously by various experimental techniques in different cuprate families [2-4]. Earlier scanning tunneling microscopy (STM) experiments reveal a checkerboard charge order on the surface of $\mathrm{Bi}_{2} \mathrm{Sr}_{2} \mathrm{CaCu}_{2} \mathrm{O}_{8+\delta}(\mathrm{Bi}-2212)$ and $\mathrm{Ca}_{2-x} \mathrm{Na}_{x} \mathrm{CuO}_{2} \mathrm{Cl}_{2}$ (Na-CCOC), especially when the superconductivity is suppressed by a magnetic field, reduced hole density, and elevated temperatures [2,5-7]. More recently, elastic and inelastic x-ray scattering experiments confirm that the charge order is a bulk phenomenon and covers a wide range of the cuprate phase diagram [8-10]. Currently, there are still heated debates regarding the nature and implications of the charge order, such as

*yayuwang@tsinghua.edu.cn

Published by the American Physical Society under the terms of the Creative Commons Attribution 4.0 International license. Further distribution of this work must maintain attribution to the author(s) and the published article's title, journal citation, and DOI. whether it is a nematic order that breaks the $C_{4}$ rotational symmetry [11-19] and whether it competes with superconductivity $[7,14,20,21]$.

Most previous STM studies on the charge order focused on underdoped and optimally doped cuprates with a welldefined pseudogap phase. It was found that the checkerboard pattern with $4 a_{0}$ periodicity ( $a_{0}$ is the lattice constant approximately $3.8 \AA$ ) is the first electronic order that emerges when doping holes into the parent Mott insulator [22], and it persists to hole density close to optimal doping [23]. With further doping into the overdoped regime, resonant x-ray scattering [9] and STM experiments [23] report the persistence of checkerboard order with increasing wavelength up to $10 a_{0}$. However, it remains unknown when and how the checkerboard order eventually vanishes in the strongly overdoped regime. Another important recent progress is the observation that the charge order is closely related to the pair density wave (PDW) order [24-29], i.e., a periodic modulation of the Cooper pairing amplitude. Both scanning Josephson tunneling microscopy on optimally doped Bi-2212 [30] and spectroscopic imaging STM on underdoped Bi-2212 [31] illustrate that the PDW has the same periodicity as the charge order, and they are positively correlated and in phase with each other. However, so far, there is no experimental study about the PDW order in the 
overdoped regime, where superconductivity is severely weakened. Therefore, it is highly desirable to elucidate the doping evolution of both the charge and pair density orders in the overdoped regime of cuprates.

In this article, we report STM studies on $\mathrm{Bi}_{2} \mathrm{Sr}_{2} \mathrm{CuO}_{6+\delta}$ (Bi-2201) cuprates with $\mathrm{La}$ or $\mathrm{Pb}$ substitutions from the optimally doped to strongly overdoped regime. We find that, with increasing hole density, the long-range checkerboard charge order gradually evolves into short-range glassy patterns consisting of diluted charge puddles. More interestingly, the unidirectional stripelike internal structure of the charge puddle pervades the entire superconducting (SC) phase and exhibit clear PDW features as revealed by the spatial variations of the SC coherence peak and gap depth. Both the charge puddles and the intrapuddle structure vanish completely in the strongly overdoped nonSC regime, when another type of short-range order with $\sqrt{2} \times \sqrt{2}$ periodicity emerges. These results shed important new light on the intricate interplay between the intertwined orders and the SC phase of cuprates.

High-quality $\mathrm{Bi}-2201$ single crystals with $\mathrm{La}$ or $\mathrm{Pb}$ substitutions are grown by the traveling solvent floating zone method and postannealed in $\mathrm{O}_{2}$ for an extended period, as described in a previous report $[32,33]$. The hole densities of the four samples studied here are $p=0.16$, $0.19,0.21$, and 0.23 , which cover the optimally doped to strongly overdoped non-SC regimes. Details about the determination of the doping level are discussed in Supplemental Material, Sec. S1 [34]. For STM experiments, the crystals are cleaved in ultrahigh vacuum at $T=77 \mathrm{~K}$ and then transferred into the STM chamber with the sample stage cooled to $T=5 \mathrm{~K}$. The STM topography is taken in the constant current mode with an electrochemically etched tungsten tip calibrated on a clean $\mathrm{Au}(111)$ surface [35], and $d I / d V$ spectra are collected by using a standard lock-in technique with a modulation bias voltage with frequency $f=423 \mathrm{~Hz}$ and $3 \mathrm{mV}$ rms amplitude.

\section{CHARGE ORDER EVOLUTION}

\section{A. Checkerboard charge order in the optimally doped sample}

We first investigate the optimally doped $\mathrm{Bi}_{2} \mathrm{Sr}_{1.63} \mathrm{La}_{0.37} \mathrm{CuO}_{6+\delta}$ with hole density $p=0.16$ and $T_{c}=32.5 \mathrm{~K}$ (denoted as OP-32K). The topographic image in Fig. 1(a) shows that the $\mathrm{Bi}$ atoms and the structural supermodulations of the exposed $\mathrm{BiO}$ surface can be clearly resolved. Figure 1(b) displays the differential conductance map $d I / d V(\boldsymbol{r}, V)$ at a representative bias voltage $V=10 \mathrm{mV}$, which directly visualizes the spatial distribution of local electron density of state (DOS) in this field of view. The most pronounced feature is the well-known checkerboard pattern, in which granular charge puddles with a typical diameter of approximately $2 \mathrm{~nm}$ form a long-range order along the $\mathrm{Cu}-\mathrm{O}$ bond direction. These results are highly consistent with previous STM studies on underdoped and optimally doped Bi-2201 [22,36].

To quantitatively characterize the checkerboard order, in Fig. 1(c), we plot the Fourier transform (FT) of the $d I / d V$ map in Fig. 1(b), which is widely utilized to determine the modulation wave vector of charge orders in cuprates $[5,7,23,37]$. The Bragg peaks of the atomic lattice are marked by solid red circles in the FT map, whereas the broad peak circled in blue indicates the wave vector $Q_{\mathrm{CO}} \approx$ $0.21\left(2 \pi / a_{0}\right)$, corresponding to a wavelength $\lambda_{\mathrm{CO}} \approx 4.8 a_{0}$ of the long-range checkerboard charge order along the $\mathrm{Cu}-\mathrm{O}$ direction. The autocorrelation map shown in Fig. 1(d) represents another powerful method to characterize the charge order, as demonstrated in Ref. [5], especially if the correlation length of the order is finite. The spatial variations of autocorrelation intensity $A C(\boldsymbol{R})$ also reveal the checkerboard order by the four bright spots circled in red, and the white dashed line is a linecut along the $\mathrm{Cu}-\mathrm{O}$ bond direction. Figure 1(e) displays the $A C(\boldsymbol{R})$ linecuts obtained at different bias voltages, where the average distance between neighboring charge puddles or the charge order wavelength $\lambda_{\mathrm{CO}}$, is indicated by the first peak. The position of the peak is nondispersive from -14 to $20 \mathrm{mV}$, demonstrating the existence of static charge order. The extracted checkerboard wavelength is $\lambda_{\mathrm{CO}} \approx 4.7 a_{0}$, which is close to $\lambda_{\mathrm{CO}} \approx 4.8 a_{0}$ obtained by the FT map in Fig. 1(c), as well as that in previous STM work $\left(\lambda_{\mathrm{CO}} \approx 5.0 a_{0}\right)$ [36] and resonant inelastic x-ray scattering $\left(\lambda_{\mathrm{CO}} \approx 4.4 a_{0}\right)$ [38] on optimally doped Bi-2201.

\section{B. Charge order evolution in the overdoped regime}

As the hole density is increased to $p=0.19,0.21$, and $0.23, T_{c}$ is decreased to 24 (OD-24K), 15 (OD-15K), and $<2 \mathrm{~K}$ (OD-0K), respectively. The charge order phenomena exhibit significant and systematic variations with doping. Figure 2(a) displays the topographic image of the OD-24K sample with chemical formula $\mathrm{Bi}_{1.62} \mathrm{~Pb}_{0.38} \mathrm{Sr}_{2} \mathrm{CuO}_{6+\delta}$, in which the surface $\mathrm{Bi}$ atoms form a regular square lattice as the structural supermodulation is removed by $\mathrm{Pb}$ substitutions [39]. Figure 2(b) is the $d I / d V$ map measured at $V=10 \mathrm{mV}$, in which granular charge puddles with a typical size of approximately $2 \mathrm{~nm}$ are still evident, but the interpuddle pattern is less close packed and less ordered than the optimally doped sample shown in Fig. 1(b). This result is directly revealed by the FT map in Fig. 2(c), in which the wave vector corresponding to the checkerboard order becomes barely visible. The $A C(\boldsymbol{R})$ map in Fig. 2(d) still has four bright spots (circled in red) but is much weaker and less symmetric than that in Fig. 1(d). By averaging the first peak position along the two directions, the interpuddle distance is obtained to be $\lambda_{\mathrm{CO}} \approx 5.4 a_{0}$. The first peak in the $A C(\boldsymbol{R})$ linecuts shown in Fig. 2(e) is nondispersive in the bias range from 0 to $30 \mathrm{mV}$, so that the observed patterns are also static charge density 

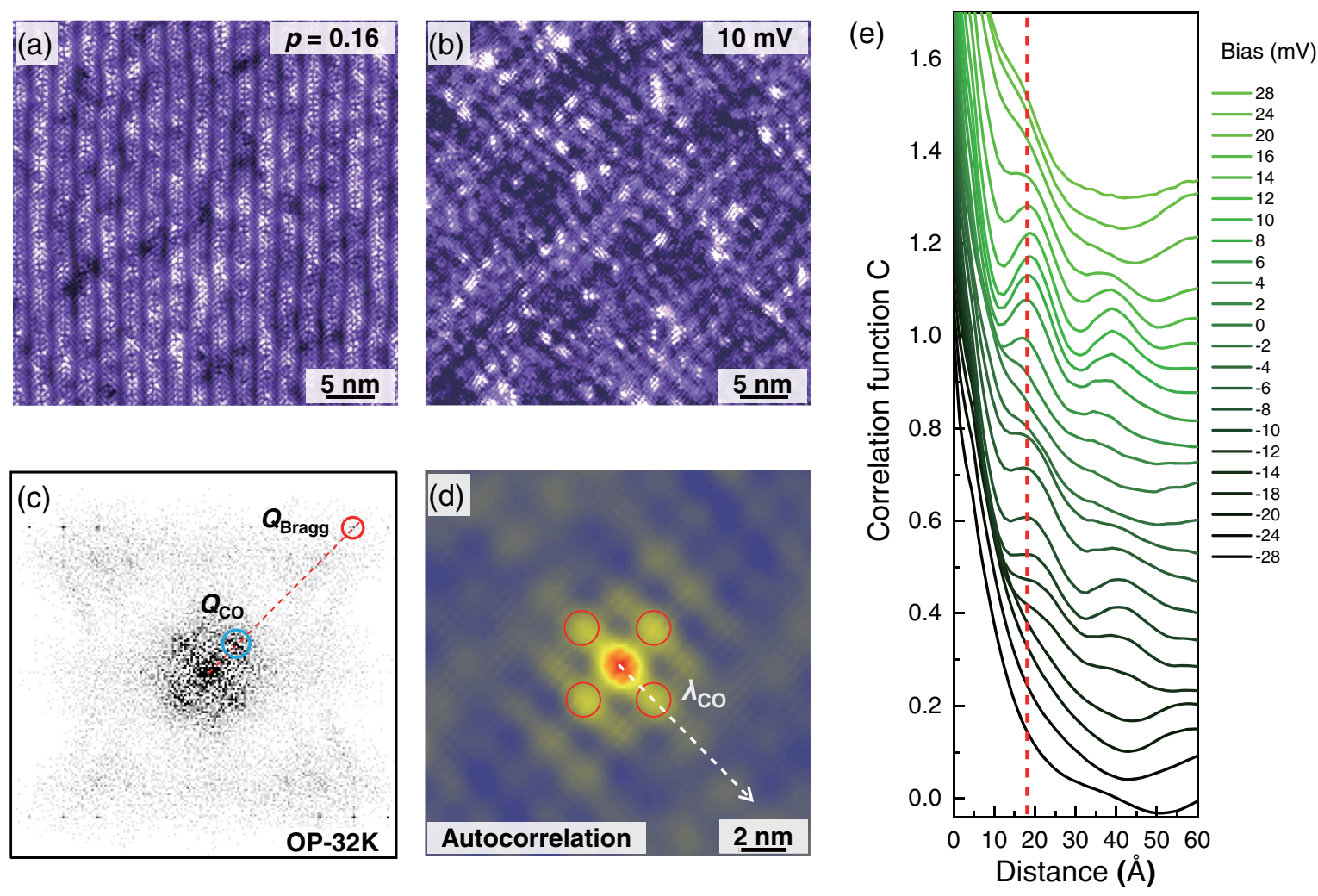

FIG. 1. The optimally doped Bi-2201 sample with $p=0.16$. (a) Atomically resolved topographic image acquired at bias voltage $V=-80 \mathrm{mV}$ and tunneling current $I=5 \mathrm{pA}$ over an area of $400 \times 400 \AA^{2}$. (b) The $d I / d V$ map measured in the same area as (a) at bias voltage $V=10 \mathrm{mV}$ and tunneling current $I=20 \mathrm{pA}$. (c) The Fourier transform map of (b), where the lattice Bragg peaks are marked by red circles and the $\mathrm{Cu}-\mathrm{O}$ bond direction is indicated by a red dashed line. The blue circle highlights the wave vector of the checkerboard charge order. (d) The autocorrelation map of (b) exhibiting a long-range checkerboard charge order, and the four red circles indicate the distance between neighboring charge puddles. The white dashed line indicates the $\mathrm{Cu}-\mathrm{O}$ bond direction, along which the autocorrelation intensity linecut is acquired. (e) Bias-dependent autocorrelation intensity $A C(\boldsymbol{R})$ linecuts. The red dashed line indicates the position of the charge order peak, which is nondispersive over a wide range of energies.

modulations with short-range correlations. These observations are not affected by the choice of setup parameters, as demonstrated in Supplemental Material, Sec. S2 [34].

In the more overdoped OD-15K sample, the checkerboard pattern is further weakened. Figures 3(a) and 3(b) are the topographic image and the $d I / d V$ map measured at $V=10 \mathrm{mV}$, respectively, in which the granular charge puddles become more diluted and are arranged in a more disordered manner. In the FT map in Fig. 3(c), the checkboard wave vector totally disappears, and the $A C(\boldsymbol{R})$ map in Fig. 3(d) has only fuzzy arcs (circled in red). The first peak in the $A C(\boldsymbol{R})$ linecuts in Fig. 3(e) is still nondispersive in the bias range from 0 to $30 \mathrm{mV}$, and the average distance between the glassy charge puddles is increased to $\lambda_{\mathrm{CO}} \approx 6.3 a_{0}$. It should be noted that the more disordered patterns at higher doping are not due to the increase of local hole density inhomogeneity. According to the normalized histograms of pseudogap size on the three SC samples (see the analysis in Supplemental Material, Sec. S3 [34]), the degree of inhomogeneity is independent of the doping level, which is the same as that in Bi-2212 [40].
When the hole density is increased further to $p=0.23$, the system enters the strongly overdoped non-SC regime. Figures 4(a) and 4(b) display the topography and $d I / d V$ map at $V=10 \mathrm{mV}$, respectively, for the OD-0K sample, where all the signatures of granular charge puddles ordered along the $\mathrm{Cu}-\mathrm{O}$ direction disappear. Interestingly, a commensurate $\sqrt{2} \times \sqrt{2}$ charge order along the nearest neighboring $\mathrm{Cu}-\mathrm{Cu}$ direction emerges, as reported previously [39]. The broken yellow squares in Fig. 4(b) indicate the small areas exhibiting the short-range $\sqrt{2} \times \sqrt{2}$ order. The FT map in Fig. 4(c) reveals rather weak and broad wave vectors (circled in blue) corresponding to this $\sqrt{2} \times \sqrt{2}$ order, which can be seen more clearly by the cross feature (circled by blue dashed lines) at the center of the $A C(\boldsymbol{R})$ map in Fig. 4(d).

The comparison between the FT map and $A C(\boldsymbol{R})$ map in the overdoped samples clearly demonstrates the advantage of the autocorrelation analysis. When the charge density modulation becomes short-ranged, its feature in the FT map becomes very fuzzy. Instead, the autocorrelation 

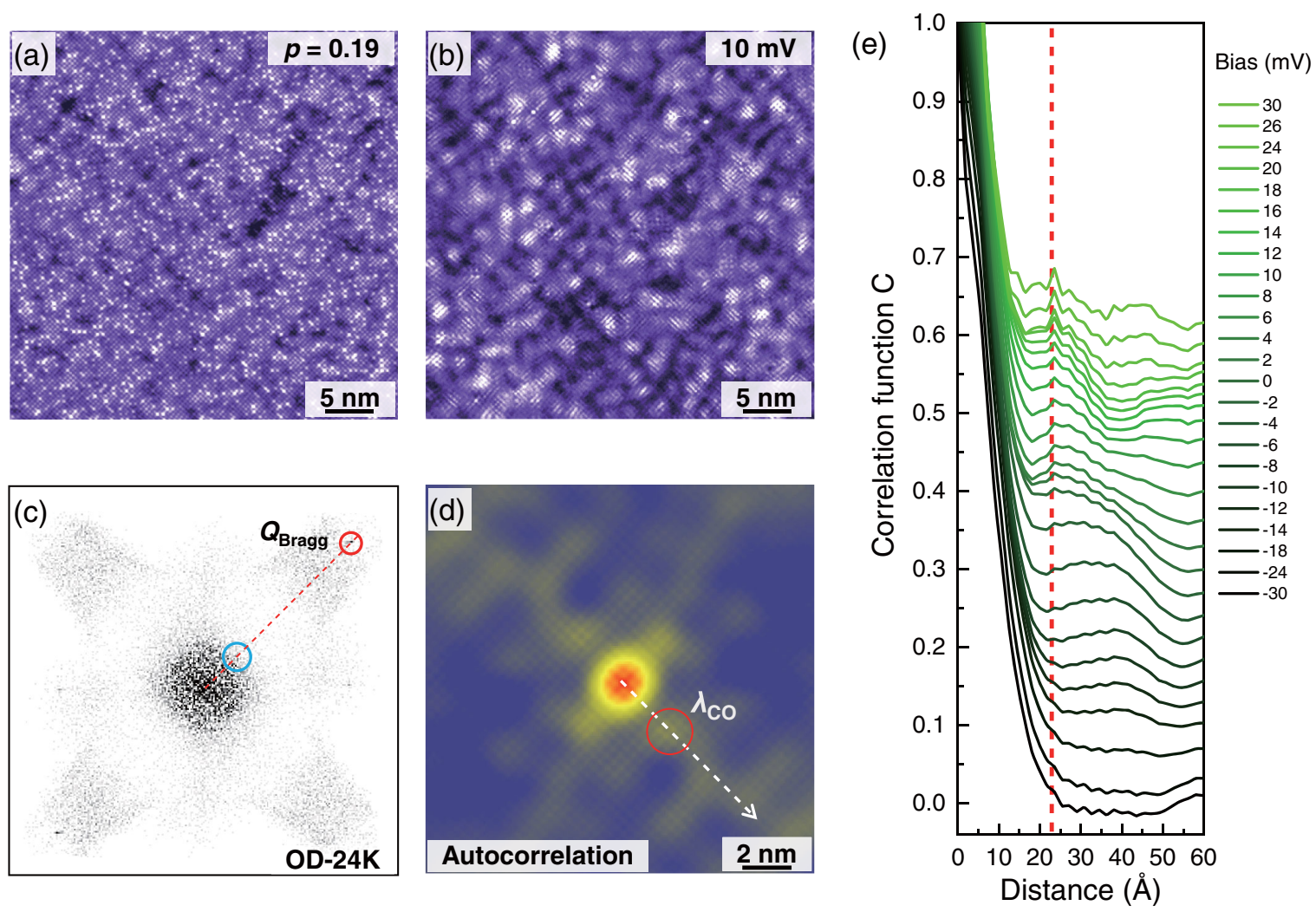

FIG. 2. The overdoped OD-24K sample with $p=0.19$. (a) Atomically resolved topographic image acquired at $V=100 \mathrm{mV}$ and $I=5 \mathrm{pA}$ over an area of $400 \times 400 \AA^{2}$. (b) The $d I / d V$ map measured in the same area as (a) at bias voltage $V=10 \mathrm{mV}$. (c) The Fourier transform map of (b), where the blue circle indicates the absence of a FT peak for the checkerboard order. (d) The autocorrelation map of (b), where the short-range charge order is revealed by four bright spots in a red circle. (e) Bias-dependent autocorrelation intensity $A C(\boldsymbol{R})$ linecuts along the $\mathrm{Cu}-\mathrm{O}$ bond direction. The red dashed line indicates the nondispersive charge order peak.

maps are more sensitive to short-range correlations; thus, the glassy patterns or small patches of orders can still be revealed.

To quantify the charge puddle density for each sample, we identify the nondispersive local maxima on $d I / d V$ maps as the center of charge puddles [indicated by yellow dots in Supplemental Material, Figs. S3(a)-S3(d) [34]] and count the total number in the measured area. Figure 5(a) indicates the hole concentrations of the samples studies here in the phase diagram, and Fig. 5(b) summarizes the doping dependence of charge puddle density, which decreases continuously with increasing hole density. On the contrary, the $\sqrt{2} \times \sqrt{2}$ charge order that prevails in the OD-0K sample displays an opposite trend. The percentage of the area with $\sqrt{2} \times \sqrt{2}$ charge order (see the estimation in Supplemental Material, Sec. S4 [34]) grows with overdoping and increases steeply upon entering the non-SC regime. This Supplemental Material section [34] also indicates that the $\sqrt{2} \times \sqrt{2}$ charge order maintains the short-range behavior in all samples and the averaged range of the order is around four periods, or $2 \mathrm{~nm}$. Therefore, in the overdoped regime of $\mathrm{Bi}-2201$, the checkerboard order consisting of granular charge puddles along the $\mathrm{Cu}-\mathrm{O}$ bond direction becomes more dilute and irregular and is eventually replaced by the $\sqrt{2} \times \sqrt{2}$ order along the $\mathrm{Cu}-\mathrm{Cu}$ direction.

Because of the orthorhombic distortion in Bi-based cuprates, a $\sqrt{2} \times \sqrt{2}$ structural modulation also exists in the surface $\mathrm{BiO}$ layer of $\mathrm{Bi}-2201$ and $\mathrm{Bi}-2212$ [41]. But this structural effect does not affect the charge order observed on the undistorted $\mathrm{CuO}_{2}$ layer due to the distinctively different characters of the two phenomena. First, the $\sqrt{2} \times \sqrt{2}$ charge order reported here is short-ranged, so its signature in the FT map is weak and broad [Fig. 4(c)]. On the contrary, the superstructure of the distorted surface $\mathrm{Bi}$ atoms is very long-ranged, and the FT peaks are as sharp as the Bragg peaks (Supplemental Material, Fig. S5 [34]). Second, the $\sqrt{2} \times \sqrt{2}$ charge order is strongly doping dependent, as shown by the masked $d I / d V$ maps in Supplemental Material, Fig. S4 [34], whereas the surface superstructure is doping independent. Third, the $\sqrt{2} \times \sqrt{2}$ charge orders demonstrated by $d I / d V$ maps exist only at low bias $|V|<30 \mathrm{meV}$, whereas the topographic images are taken at high biases. Therefore, the $\sqrt{2} \times \sqrt{2}$ charge order is an electronic effect without structural origin. 

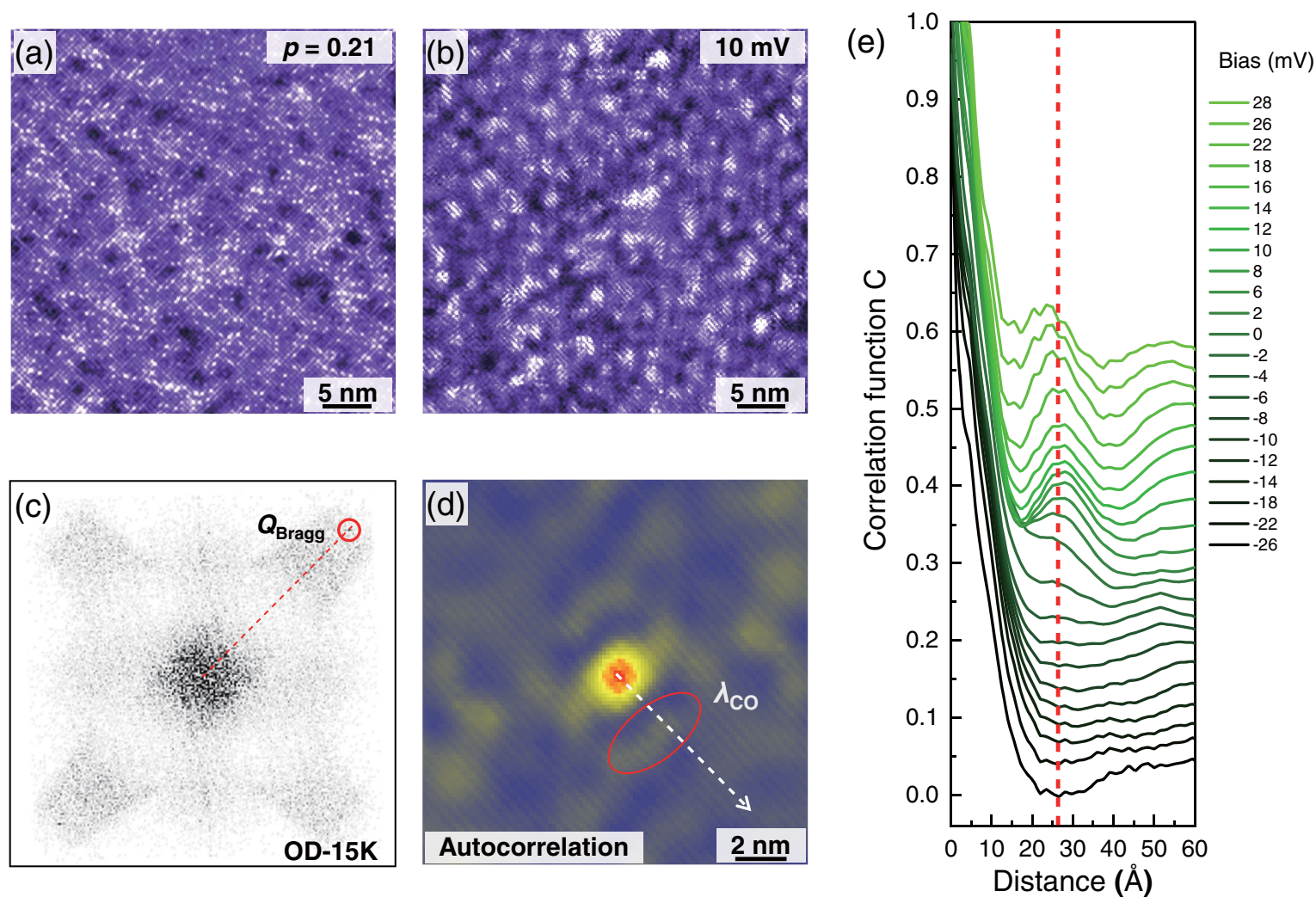

FIG. 3. The overdoped OD-15K sample with $p=0.21$. (a) Atomically resolved topographic image acquired at $V=-100 \mathrm{mV}$ and $I=20 \mathrm{pA}$ over an area of $400 \times 400 \AA^{2}$. (b) The $d I / d V$ map measured in the same area as (a) at bias voltage $V=10 \mathrm{mV}$. (c) The Fourier transform map of (b). (d) The autocorrelation map of (b), where the bright arc marked by the red oval indicates the remnant charge order. (e) Bias-dependent autocorrelation intensity $A C(\boldsymbol{R})$ linecuts. The nondispersive first peaks suggest that the fussy arc corresponds to the glassy patterns of charge puddles.

\section{The internal structure of charge puddles}

Another intriguing observation is that, in the $d I / d V$ maps of all three SC samples shown in Figs. 1(b), 2(b), and 3(b), there are bright stripes within each charge puddle pointing to either of the two orthogonal directions. This internal electronic structure is commensurate with the underlying $\mathrm{Cu}$ lattice but breaks the $C_{4}$ rotational symmetry of the $\mathrm{O}_{x}-\mathrm{O}_{y}$ bonds within a $\mathrm{CuO}_{2}$ unit cell. These features are consistent with the nematic order reported before by STM studies in various cuprates $[18,42-44]$. In the OD$15 \mathrm{~K}$ sample, the stripy pattern is still so pronounced that it can be directly visualized by the mazelike topography in
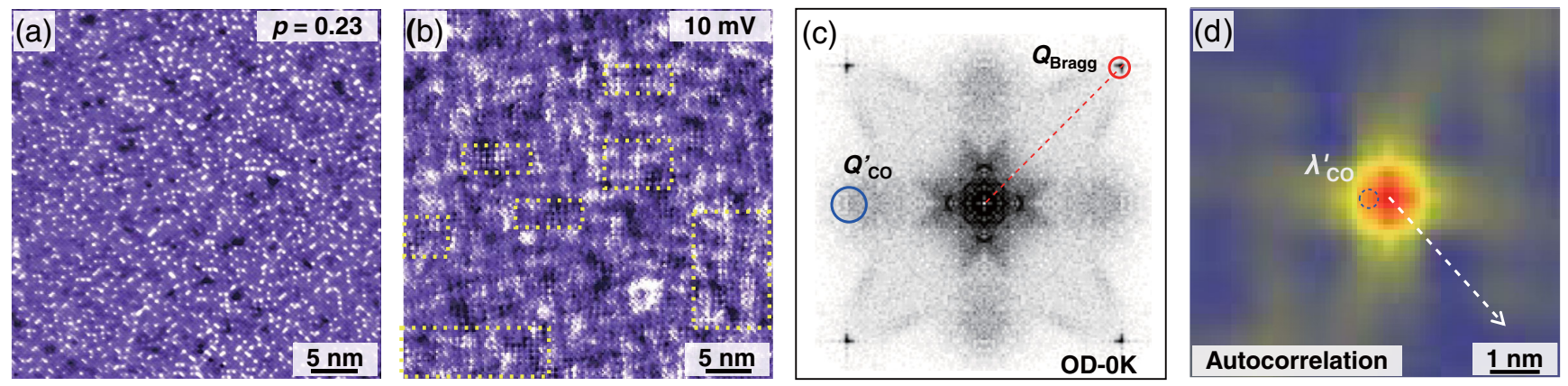

FIG. 4. The overdoped OD-0K sample with $p=0.23$. (a) Atomically resolved topographic image acquired at $V=100 \mathrm{mV}$ and $I=5 \mathrm{pA}$ over an area of $350 \times 350 \AA^{2}$. (b) The $d I / d V$ map measured in the same area as (a) at bias voltage $V=10 \mathrm{mV}$. The patches of short-range $\sqrt{2} \times \sqrt{2}$ order are indicated by the broken yellow squares. (c) The Fourier transform map of (b) displaying the Bragg peaks in the red circles, as well as the short-range $\sqrt{2} \times \sqrt{2}$ charge order in the blue circle. (d) The autocorrelation map of (b), where the central cross and the spot in the circle indicate the short-range $\sqrt{2} \times \sqrt{2}$ charge order in separated patches. 


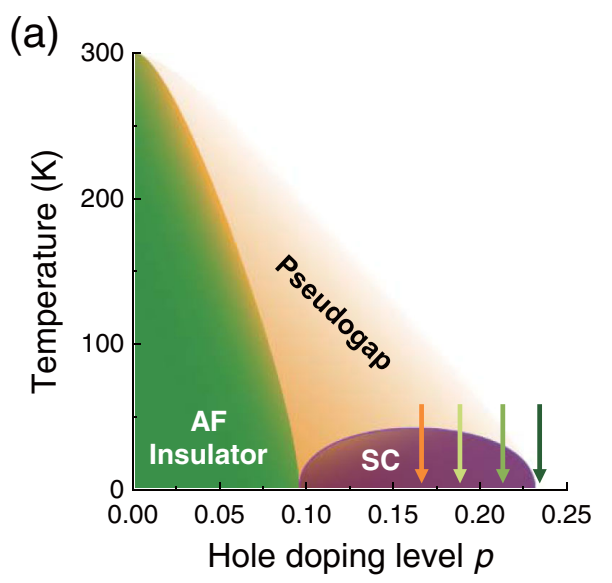

(c)

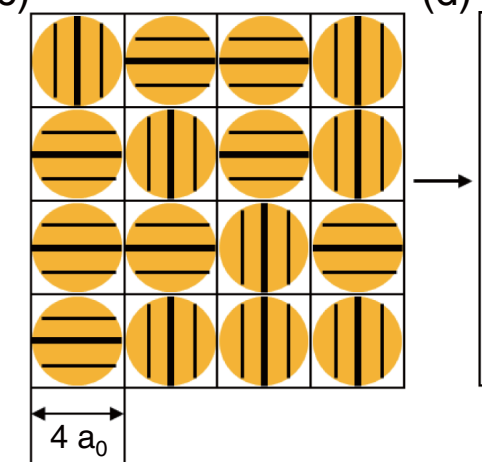

(b)

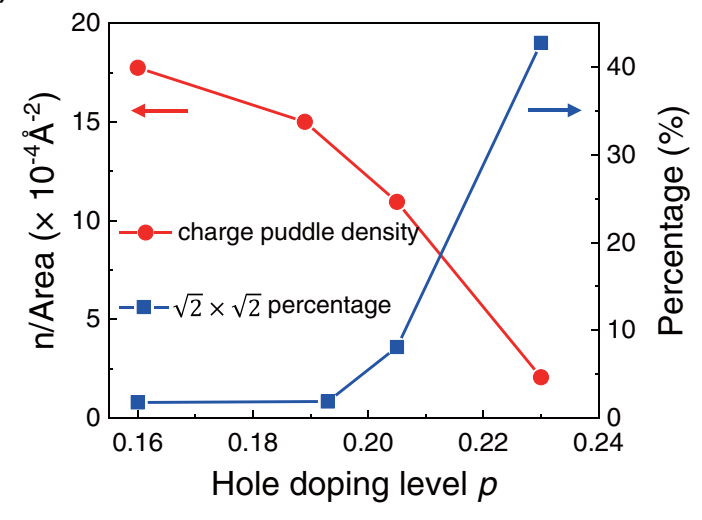

(e)
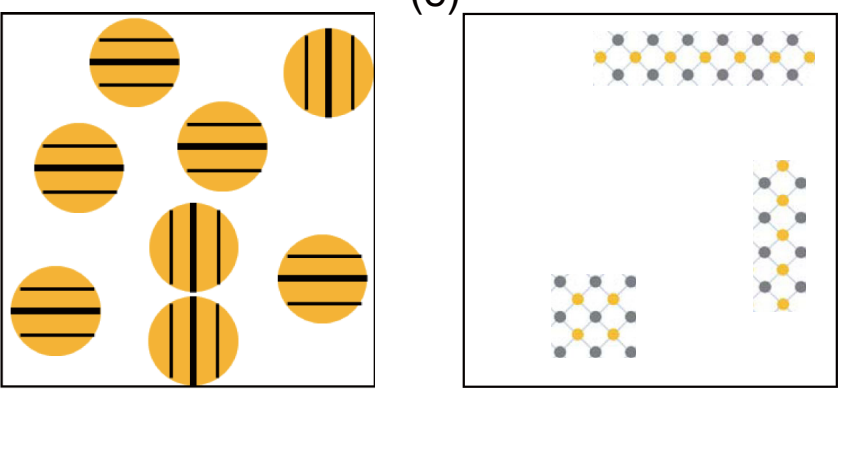

FIG. 5. The doping evolution of charge orders. (a) The schematic electronic phase diagram of Bi-2201, and the arrows indicate the hole densities of the four samples studied in this work. (b) Doping dependence of the charge puddle density and the percentage of areas with $\sqrt{2} \times \sqrt{2}$ charge order. (c)-(e) Schematic cartoons illustrating the evolution of charge order with increasing doping. The yellow spheres represent the charge puddles with a size of approximately $4 a_{0}$ and the black lines sketch the internal stripy structure. In the overdoped non-SC sample, the charge puddles with stripy internal structure disappear, and small patches with $\sqrt{2} \times \sqrt{2}$ charge order emerge.

Fig. 3(a), also similar to that observed previously in lightly doped CCOC [16]. The granular charge puddles and the constituent stripes disappear altogether in the overdoped non-SC sample OD-OK [Fig. 4(b)].

\section{PAIR DENSITY MODULATIONS}

\section{A. Pair density wave in the optimally doped regime}

The evolution of charge order is also closely entangled with the PDW order, which has become a focus topic in the debates of intertwined orders in cuprates [24-31,45,46]. Traditionally, the charge order is revealed by the periodic modulation of $d I / d V$ at specific energies, as shown above in the $d I / d V$ maps. In order to unveil the PDW order, one must extract the spectral information directly related to the SC properties. In a previous report [31], we demonstrate that there are two quantities in the $d I / d V$ spectrum that directly characterize the strength of local superconductivity. The first is the depth of the SC gap, which reflects the depletion of low-energy quasiparticle spectral weight by Cooper pairing. This quantity can be represented by the height difference $H=d I / d V\left(V_{\mathrm{SC}}\right)-d I / d V(0)$ between the $d I / d V$ values at the SC gap edge and zero bias. The second is the amplitude of the SC coherence peak. This quantity can be characterized by the minus second derivative $D(V)=-d^{3} I / d V^{3}$ at the SC gap edge of the $d I / d V$ spectrum, which is proportional to the sharpness of the coherence peak. The positive correlation between the SC coherence peak and the strength of local superconductivity in cuprates is an empirical observation that is confirmed by various independent experiments. For the samples with different hole concentrations, the SC coherence peak is enhanced when approaching the optimal doping [47,48]. On a local scale, the SC coherence peak is suppressed with impurities [49], magnetic field [50,51], and increasing temperature [52].

More recently, a combined STM and scanning Josephson tunneling experiment has been performed on the iron-based superconductor [53], another unconventional superconductor with spatially inhomogeneous superfluid density. It clearly shows that the height and sharpness of the SC coherence peak detected by single-particle tunneling has a 

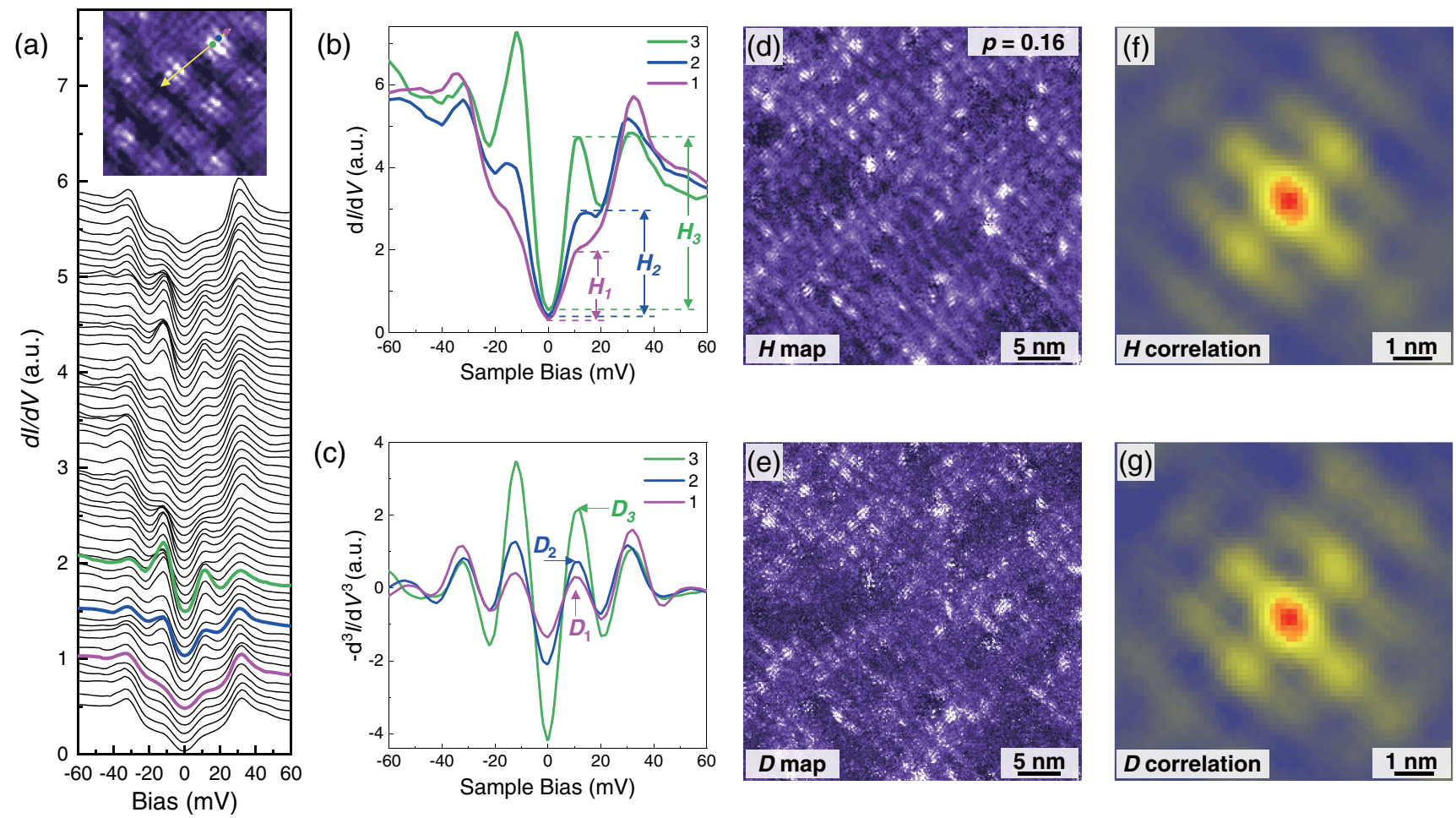

FIG. 6. The PDW features in the optimally doped sample. (a) The spectra taken along the yellow arrow in the inset $d I / d V$ map. (b) Three representative curves taken on the colored spots in (a), and the gap depth $H$ of each curve is illustrated by the dashed line. (c) The $D(V)=-d^{3} I / d V^{3}$ curves of the three curves in (b). The $D\left(V_{\mathrm{sc}}\right)$ value at $V_{\mathrm{sc}}(\boldsymbol{r}) \sim 10 \mathrm{mV}$ shows the sharpness of the local SC coherence peak. (d) The $H$ map exhibits the distribution of local gap depth $H(\boldsymbol{r})$. (e) The $D$ map displays the spatial distribution of SC coherence peak sharpness at each location. (f),(g) The intensity distribution of cross-correlation $C(\boldsymbol{R})$ between the $H$ map and $D$ map with the $d I / d V(10 \mathrm{mV}, \boldsymbol{r})$ map in Fig. 1(b). The maximum value at the center indicates a positive correlation.

strong positive correlation to the superfluid density detected by Josephson tunneling at the same location with atomic resolution. This remarkable experimental achievement unambiguously justifies our data analysis method, which reveals similar PDW features to that obtained by scanning Josephson tunneling microscopy on Bi-2212 [30].

We first apply these spectral analyses to the optimally doped Bi-2201 sample. Figure 6(a) displays $d I / d V$ curves along a line in OP-32K, which crosses two charge puddles as indicated in the inset. The high-quality spectra are individual $d I / d V$ curves from maps taken over a smaller area $\left(180 \times 180 \AA^{2}\right)$ with a larger setting current $(I=200 \mathrm{pA})$ and longer integration time. All the spectra possess two separated gaps: a large pseudogap with typical size $\Delta_{\mathrm{PG}} \sim 40 \mathrm{meV}$ and a small SC gap $\Delta_{\mathrm{SC}} \sim 10 \mathrm{meV}$. Even from the raw data, it can be easily seen that the SC coherence peaks manifest spatial modulations following the charge order and are much more pronounced on the granular puddles. Three representative $d I / d V$ spectra are shown in Fig. 6(b) with the corresponding colored spots marked in the inset. The spectrum taken on top of the bright stripe within a puddle (green curve) has very sharp SC coherence peaks. The blue curve taken on a less bright stripe has relatively weaker coherence peaks, whereas the magenta curve taken between two puddles has shoulders instead of peaks at $V_{\mathrm{SC}}= \pm 10 \mathrm{mV}$. The depth of the SC gap as represented by the height difference $H=$ $d I / d V\left(V_{\mathrm{SC}}\right)-d I / d V(0)$ for the three curves are marked by the dashed lines in Fig. 6(b), which clearly reveals that the green, blue, and magenta curves have the strongest, intermediate, and weakest superconductivity, respectively. Figure 6(c) exhibits the $D(V)=-d^{3} I / d V^{3}$ curves for the three curves, which directly reveals that the green, blue, and magenta curves have the strongest, intermediate, and weakest local superfluid density, respectively. These two different analysis methods thus give the same conclusion regarding the spatial variations of the strength of superconductivity, although the gap sizes are almost the same.

To directly visualize the spatial distribution of pair density, the SC gap depth $H$ and coherence peak sharpness $D$ are extracted from each $d I / d V$ curve acquired on the area in Fig. 1(a), and the $H$ map and $D$ map at $V_{\mathrm{SC}} \sim$ $10 \mathrm{mV}$ are displayed in Figs. 6(d) and 6(e), respectively. Both images reveal similar long-range checkerboardlike patterns, which unveil the periodic modulation of SC properties, i.e., a well-defined PDW order. More interestingly, in both images, the pair density modulation also has the stripelike internal structure within each puddle, which 
can already be anticipated from the linecut in Fig. 6(a). To elucidate the relationship between charge and pair density modulations, the cross-correlations of $H(\boldsymbol{r})$ and $D\left(\boldsymbol{r}, V_{\mathrm{SC}}\right)$ with the corresponding $d I / d V(\boldsymbol{r}, V=10 \mathrm{mV})$ are shown in Figs. 6(f) and 6(g). Both cross-correlation maps exhibit a maximum at the center, indicating a positive correlation between the charge and pair density modulations.

\section{B. Pair density modulations in the overdoped regime}

We extend the same measurement and analysis procedures regarding the pair density modulations to the overdoped OD-24K sample with $p=0.19$. In the inset in Fig. 7(a), a linecut of $d I / d V$ curves is obtained across a single puddle. For each curve, there are still well-defined SC coherence peaks with gap size $\Delta_{\mathrm{SC}} \sim 10 \mathrm{meV}$, while the pseudogap size is reduced to $\Delta_{\mathrm{PG}} \sim 25 \mathrm{meV}$ and the features become much weaker. Similar to that in the OP-32K sample, the SC coherence peaks exhibit periodic spatial variations that are strongly correlated to the charge puddle and the stripelike internal structure. The three representative colored curves in Fig. 7(a) demonstrate that the SC coherence peaks are most pronounced at the bright stripe within a puddle (green curve), become slightly weakened (blue curve) at a less-bright stripe, and evolve into broad shoulders at locations between charge puddles (magenta curve). The $H$ map and $D$ map of the OD-24K sample on the same area as Fig. 2(a) are depicted in
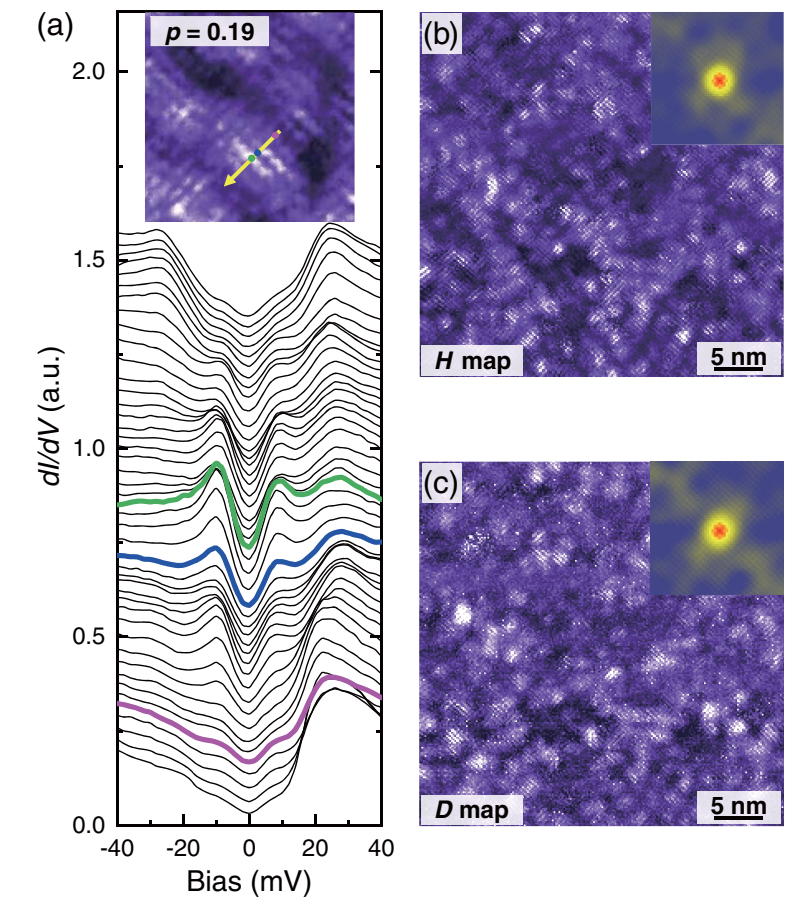

Figs. 7(b) and 7(c), respectively. Despite the absence of long-range checkerboard order, the periodic distribution of local SC strength still has a short-range PDW pattern and pronounced stripelike internal structure. The insets in Figs. 7(b) and 7(c) illustrate the cross-correlation between the $H$ map and $D$ map with the $d I / d V$ map in Fig. 2(b), and the maximum at the center demonstrates that the pair density modulations are positively correlated with the charge density modulations.

When the hole density is increased to $p=0.21$, the pseudogap of the OD-15K sample becomes too small to be distinguished from the SC gap, as revealed by the $d I / d V$ curves in Fig. 7(d). Nevertheless, the SC coherence peak features still closely follow the granular puddles and stripes, as revealed by the three representative colored curves in the linecut. Even though the long-range interpuddle ordering disappears, the local puddles and internal stripes are still evident in both the $H$ map [Fig. 7(e)] and $D$ map [Fig. 7(f)]. The insets display the cross-correlation maps between them and the charge order map in Fig. 3(b), and the central maximum reveals a positive correlation between the intertwined pair and charge density modulations.

\section{DISCUSSION}

The origin of the various intertwined orders in cuprates, the intricate relationship between them, and implications to superconductivity have been core issues
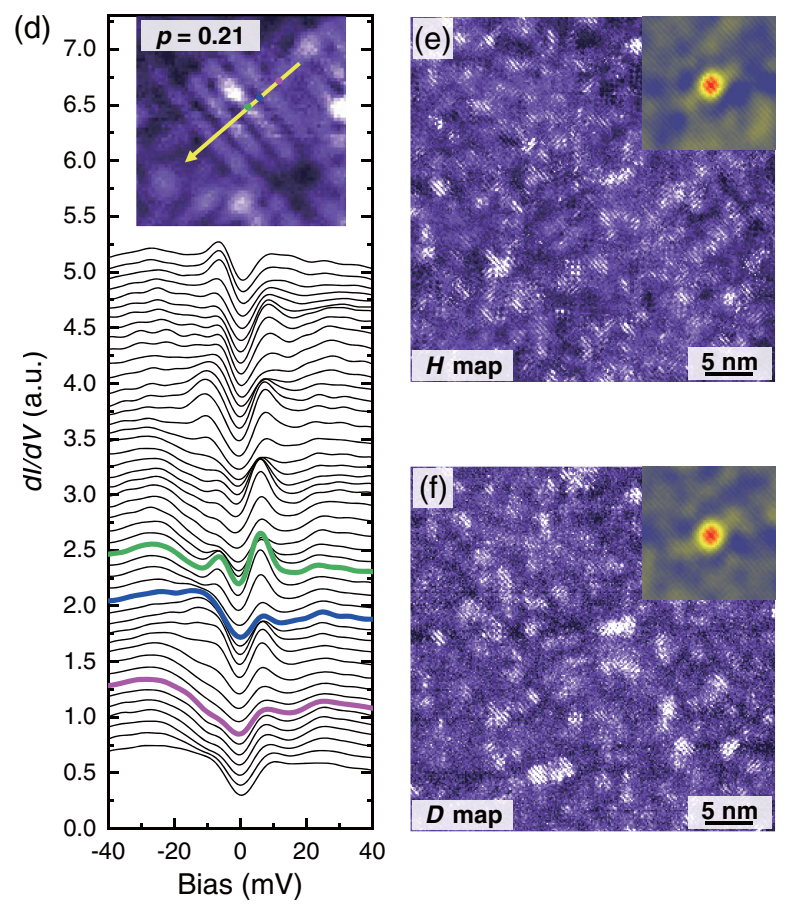

FIG. 7. The pair density modulation features in two overdoped SC samples. (a),(d) Spectral linecuts along the yellow arrows on the OD-24K and OD-15K samples, respectively. The insets are the corresponding $d I / d V$ maps $\left(50 \times 50 \AA^{2}\right)$ taken at bias voltage $V=10 \mathrm{mV}$. (b),(e) The $H$ maps of the OD-24K and OD-15K samples, and the insets are the same cross-correlation plots as in Fig. 6(f). (c),(f) The $D$ maps of the OD-24K and OD-15K samples, and the corresponding cross-correlations are shown in the insets. The maximum at the center indicates a positive correlation between the charge and pair density modulations. 
in the mechanism problem. Our systematic STM studies on Bi-2201 cover a broad range of phase diagrams from the optimally doped to overdoped non-SC regimes [Fig. 5(a)], which is much less explored compared to the underdoped regime. In particular, the quantitative analysis of the overall $d I / d V$ line shape enables us to extract the characteristic features that are selectively sensitive to local superconductivity, and the correlation analysis between various spatial patterns reveals the relationship between the intertwined orders. Our results clarify several important issues concerning the fate of charge and pair density modulations in overdoped cuprates, as discussed below.

First, we show that, with increasing doping, the longrange checkerboard orders in the underdoped and optimally samples gradually evolve into a glassy state with diluted and disordered charge puddles in the overdoped regime. This picture is distinctively different from the scenario of expanded checkerboard wavelength with overdoping while keeping the ordered structure intact $[9,23]$, despite the deceptive agreement of increased average distance between neighboring puddles in both pictures. The interpretation for the superlattice expansion involves the weak coupling theory of Fermi surface nesting with an enlarged hole pocket $[4,36]$, whereas our results indicate that the strong local interaction is still essential even in the overdoped regime.

Second, we show that the nematic electronic structure is highly robust against overdoping and exists in all SC samples studied so far in the Bi-2201 system. As illustrated by the cartoon in Fig. $5(\mathrm{c})$, the $C_{4}$-symmetry-breaking stripes form a charge puddle with a size of approximately $4 a_{0}$, or $2 \mathrm{~nm}$, and the puddles are subsequently organized into long-range checkerboard order in the optimally doped sample and diluted glassy state in the overdoped regime [Fig. 5(d)]. This observation provides another evidence for the relevance of the residual correlation effect between doped holes in overdoped cuprates [11,15,18,54-56].

Third, we show that the charge and pair density modulations are closely entangled and coexist with the SC phases at least up to $p=0.21$. More importantly, they vanish altogether in the strongly overdoped limit when superconductivity disappears. This observation suggests that these incipient charge and pair density modulations are not simple competing orders to superconductivity but may instead share some common origins with Cooper pairing. In the strongly overdoped non-SC regime, they are eventually replaced by the $\sqrt{2} \times \sqrt{2}$ charge order [Fig. 5(e)], which may represent a mechanism for suppressing Cooper pairing [38].

In summary, our STM studies on Bi-2201 cuprates reveal the evolution of charge and pair density modulations in the overdoped regime. We find a gradual deformation of the checkerboard charge order, but the granular puddles with internal nematicity are more robust and exhibit close correlations with local pair density modulations. The dichotomy between the intrapuddle structure and interpuddle order, plus the complete disappearance of both features in the non-SC regime, sheds important new light on the nature of these intertwined orders in cuprates.

\section{ACKNOWLEDGMENTS}

This work was supported by the MOST of China Grant No. 2017YFA0302900, NSFC Grant No. 11534007, MOST of China Grant No. 2016YFA0300300, and the Strategic Priority Research Program (B) of the Chinese Academy of Sciences (XDB25000000). This work is supported in part by the Beijing Advanced Innovation Center for Future Chip (ICFC).

[1] B. Keimer, S. A. Kivelson, M. R. Norman, S. Uchida, and J. Zaanen, From Quantum Matter to High-Temperature Superconductivity in Copper Oxides, Nature (London) $\mathbf{5 1 8}$, 179 (2015).

[2] J. E. Hoffman, E. W. Hudson, K. M. Lang, V. Madhavan, H. Eisaki, S. Uchida, and J. C. Davis, A Four Unit Cell Periodic Pattern of Quasi-Particle States Surrounding Vortex Cores in $\mathrm{Bi}_{2} \mathrm{Sr}_{2} \mathrm{CaCu}_{2} \mathrm{O}_{8+\delta}$, Science 295, 466 (2002).

[3] M. Hücker, N. B. Christensen, A. T. Holmes, E. Blackburn, E. M. Forgan, R. Liang, D. A. Bonn, W. N. Hardy, O. Gutowski, M. v. Zimmermann, S. M. Hayden, and J. Chang, Competing Charge, Spin, and Superconducting Orders in Underdoped $\mathrm{YBa}_{2} \mathrm{Cu}_{3} \mathrm{O}_{y}$, Phys. Rev. B 90, 054514 (2014).

[4] R. Comin, A. Frano, M. M. Yee, Y. Yoshida, H. Eisaki, E. Schierle, E. Weschke, R. Sutarto, F. He, A. Soumyanarayanan, Y. He, M. Le Tacon, I. S. Elfimov, J. E. Hoffman, G. A. Sawatzky, B. Keimer, and A. Damascelli, Charge Order Driven by Fermi-Arc Instability in $\mathrm{Bi}_{2} \mathrm{Sr}_{2-x} \mathrm{La}_{x} \mathrm{CuO}_{6+\delta}$, Science 343, 390 (2014).

[5] T. Hanaguri, C. Lupien, Y. Kohsaka, D. H. Lee, M. Azuma, M. Takano, H. Takagi, and J. C. Davis, A 'Checkerboard' Electronic Crystal State in Lightly Hole-Doped $\mathrm{Ca}_{2-x} \mathrm{Na}_{x} \mathrm{CuO}_{2} \mathrm{Cl}_{2}$, Nature (London) 430, 1001 (2004).

[6] Y. Kohsaka, C. Taylor, K. Fujita, A. Schmidt, C. Lupien, T. Hanaguri, M. Azuma, M. Takano, H. Eisaki, H. Takagi, S. Uchida, and J. C. Davis, An Intrinsic Bond-Centered Electronic Glass with Unidirectional Domains in Underdoped Cuprates, Science 315, 1380 (2007).

[7] E. H. D. Neto, P. Aynajian, A. Frano, R. Comin, E. Schierle, E. Weschke, A. Gyenis, J. S. Wen, J. Schneeloch, Z. J. Xu, S. Ono, G. D. Gu, M. Le Tacon, and A. Yazdani, Ubiquitous Interplay between Charge Ordering and High-Temperature Superconductivity in Cuprates, Science 343, 393 (2014).

[8] O. J. Lipscombe, S. M. Hayden, B. Vignolle, D. F. McMorrow, and T.G. Perring, Persistence of High-Frequency Spin Fluctuations in Overdoped Superconducting $\mathrm{La}_{2-x} \mathrm{Sr}_{x} \mathrm{CuO}_{4}$ $(x=0.22)$, Phys. Rev. Lett. 99, 067002 (2007).

[9] Y. Y. Peng, R. Fumagalli, Y. Ding, M. Minola, S. Caprara, D. Betto, M. Bluschke, G. M. De Luca, K. Kummer, E. Lefrancois, M. Salluzzo, H. Suzuki, M. Le Tacon, X. J. Zhou, N. B. Brookes, B. Keimer, L. Braicovich, M. Grilli, and G. Ghiringhelli, Re-entrant Charge Order in Overdoped $(\mathrm{Bi}, \mathrm{Pb})_{2.12} \mathrm{Sr}_{1.88} \mathrm{CuO}_{6+\delta}$ outside the Pseudogap Regime, Nat. Mater. 17, 697 (2018). 
[10] R. Arpaia, S. Caprara, R. Fumagalli, G. De Vecchi, Y. Y. Peng, E. Andersson, D. Betto, G. M. De Luca, N. B. Brookes, F. Lombardi, M. Salluzzo, L. Braicovich, C. Di Castro, M. Grilli, and G. Ghiringhelli, Dynamical Charge Density Fluctuations Pervading the Phase Diagram of a Cu-Based High- $T_{c}$ Superconductor, Science 365, 906 (2019).

[11] J. Zaanen and O. Gunnarsson, Charged Magnetic Domain Lines and the Magnetism of High-T $T_{c}$ Oxides, Phys. Rev. B 40, 7391 (1989).

[12] J. Zaanen and A. M. Oles, Striped Phase in the Cuprates as a Semiclassical Phenomenon, Ann. Phys. (Berlin) 508, 224 (1996).

[13] S. A. Kivelson, I. P. Bindloss, E. Fradkin, V. Oganesyan, J. M. Tranquada, A. Kapitulnik, and C. Howald, How to Detect Fluctuating Stripes in the High-Temperature Superconductors, Rev. Mod. Phys. 75, 1201 (2003).

[14] E. Berg, E. Fradkin, S. A. Kivelson, and J. M. Tranquada, Striped Superconductors: How Spin, Charge and Superconducting Orders Intertwine in the Cuprates, New J. Phys. 11, 115004 (2009).

[15] M. J. Lawler, K. Fujita, J. Lee, A. R. Schmidt, Y. Kohsaka, C. K. Kim, H. Eisaki, S. Uchida, J. C. Davis, J. P. Sethna, and E. A. Kim, Intra-Unit-Cell Electronic Nematicity of the High- $T_{c}$ Copper-Oxide Pseudogap States, Nature (London) 466, 347 (2010).

[16] Y. Kohsaka, T. Hanaguri, M. Azuma, M. Takano, J. C. Davis, and H. Takagi, Visualization of the Emergence of the Pseudogap State and the Evolution to Superconductivity in a Lightly Hole-Doped Mott Insulator, Nat. Phys. 8, 534 (2012).

[17] L. M. Nie, G. Tarjus, and S. A. Kivelson, Quenched Disorder and Vestigial Nematicity in the Pseudogap Regime of the Cuprates, Proc. Natl. Acad. Sci. U.S.A. 111, 7980 (2014).

[18] Y. Zheng, Y. Fei, K. Bu, W. Zhang, Y. Ding, X. Zhou, J. E. Hoffman, and Y. Yin, The Study of Electronic Nematicity in an Overdoped $(\mathrm{Bi}, \mathrm{Pb})_{2} \mathrm{Sr}_{2} \mathrm{CuO}_{6+\delta}$ Superconductor Using Scanning Tunneling Spectroscopy, Sci. Rep. 7, 8059 (2017).

[19] S. Mukhopadhyay, R. Sharma, C. K. Kim, S. D. Edkins, M. H. Hamidian, H. Eisaki, S. Uchida, E. A. Kim, M. J. Lawler, A. P. Mackenzie, J. C. S. Davis, and K. Fujita, Evidence for a Vestigial Nematic State in the Cuprate Pseudogap Phase, Proc. Natl. Acad. Sci. U.S.A. 116, 13249 (2019).

[20] J. M. Tranquada, B. J. Sternlieb, J. D. Axe, Y. Nakamura, and S. Uchida, Evidence for Stripe Correlations of Spins and Holes in Copper-Oxide Superconductors, Nature (London) 375, 561 (1995).

[21] J. Chang, E. Blackburn, A. T. Holmes, N. B. Christensen, J. Larsen, J. Mesot, R. X. Liang, D. A. Bonn, W. N. Hardy, A. Watenphul, M. von Zimmermann, E. M. Forgan, and S. M. Hayden, Direct Observation of Competition between Superconductivity and Charge Density Wave Order in $\mathrm{YBa}_{2} \mathrm{Cu}_{3} \mathrm{O}_{6.67}$, Nat. Phys. 8, 871 (2012).

[22] P. Cai, W. Ruan, Y. Peng, C. Ye, X. Li, Z. Hao, X. Zhou, D.-H. Lee, and Y. Wang, Visualizing the Evolution from the Mott Insulator to a Charge-Ordered Insulator in Lightly Doped Cuprates, Nat. Phys. 12, 1047 (2016).
[23] T. A. Webb, M. C. Boyer, Y. Yin, D. Chowdhury, Y. He, T. Kondo, T. Takeuchi, H. Ikuta, E. W. Hudson, J. E. Hoffman, and M. H. Hamidian, Density Wave Probes Cuprate Quantum Phase Transition, Phys. Rev. X 9, 021021 (2019).

[24] H. D. Chen, O. Vafek, A. Yazdani, and S. C. Zhang, Pair Density Wave in the Pseudogap State of High Temperature Superconductors, Phys. Rev. Lett. 93, 187002 (2004).

[25] P. A. Lee, Amperean Pairing and the Pseudogap Phase of Cuprate Superconductors, Phys. Rev. X 4, 031017 (2014).

[26] Y. X. Wang, D. F. Agterberg, and A. Chubukov, Coexistence of Charge-Density-Wave and Pair-Density-Wave Orders in Underdoped Cuprates, Phys. Rev. Lett. 114, 197001 (2015).

[27] W. L. Tu and T. K. Lee, Genesis of Charge Orders in High Temperature Superconductors, Sci. Rep. 6, 18675 (2016).

[28] R. G. Cai, L. Li, Y. Q. Wang, and J. Zaanen, Intertwined Order and Holography: The Case of Parity Breaking Pair Density Waves, Phys. Rev. Lett. 119, 181601 (2017).

[29] Z. H. Dai, Y. H. Zhang, T. Senthil, and P. A. Lee, PairDensity Waves, Charge-Density Waves, and Vortices in High-T $T_{c}$ Cuprates, Phys. Rev. B 97, 174511 (2018).

[30] M. H. Hamidian, S. D. Edkins, S. H. Joo, A. Kostin, H. Eisaki, S. Uchida, M. J. Lawler, E. A. Kim, A. P. Mackenzie, K. Fujita, J. Lee, and J. C. S. Davis, Detection of a CooperPair Density Wave in $\mathrm{Bi}_{2} \mathrm{Sr}_{2} \mathrm{CaCu}_{2} \mathrm{O}_{8+x}$, Nature (London) 532, 343 (2016).

[31] W. Ruan, X. T. Li, C. Hu, Z. Q. Hao, H. W. Li, P. Cai, X. J. Zhou, D. H. Lee, and Y. Y. Wang, Visualization of the Periodic Modulation of Cooper Pairing in a Cuprate Superconductor, Nat. Phys. 14, 1178 (2018).

[32] Y. Peng, J. Meng, D. Mou, J. He, L. Zhao, Y. Wu, G. Liu, X. Dong, S. He, J. Zhang, X. Wang, Q. Peng, Z. Wang, S. Zhang, F. Yang, C. Chen, Z. Xu, T. K. Lee, and X. J. Zhou, Disappearance of Nodal Gap across the InsulatorSuperconductor Transition in a Copper-Oxide Superconductor, Nat. Commun. 4, 2459 (2013).

[33] L. Zhao, W. T. Zhang, H. Y. Liu, J. Q. Meng, G. D. Liu, W. Lu, X. L. Dong, and X. J. Zhou, High-Quality Large-Sized Single Crystals of Pb-Doped $\mathrm{Bi}_{2} \mathrm{Sr}_{2} \mathrm{CuO}_{6+\delta}$ High-T $T_{c}$ Superconductors Grown with Traveling Solvent Floating Zone Method, Chin. Phys. Lett. 27 (2010).

[34] See Supplemental Material at http://link.aps.org/ supplemental/10.1103/PhysRevX.11.011007 for additional information.

[35] C. Ye, P. Cai, R. Yu, X. Zhou, W. Ruan, Q. Liu, C. Jin, and Y. Wang, Visualizing the Atomic-Scale Electronic Structure of the Ca2CuO2Cl2 Mott Insulator, Nat. Commun. 4, 1365 (2013).

[36] W. D. Wise, M. C. Boyer, K. Chatterjee, T. Kondo, T. Takeuchi, H. Ikuta, Y. Wang, and E. W. Hudson, ChargeDensity-Wave Origin of Cuprate Checkerboard Visualized by Scanning Tunnelling Microscopy, Nat. Phys. 4, 696 (2008).

[37] W. D. Wise, K. Chatterjee, M. C. Boyer, T. Kondo, T. Takeuchi, H. Ikuta, Z. Xu, J. Wen, G. D. Gu, Y. Wang, and E. W. Hudson, Imaging Nanoscale Fermi-Surface Variations in an Inhomogeneous Superconductor, Nat. Phys. 5, 213 (2009).

[38] Y. Y. Peng, M. Salluzzo, X. Sun, A. Ponti, D. Betto, A. M. Ferretti, F. Fumagalli, K. Kummer, M. Le Tacon, X. J. Zhou, 
N. B. Brookes, L. Braicovich, and G. Ghiringhelli, Direct Observation of Charge Order in Underdoped and Optimally Doped $\mathrm{Bi}_{2}(\mathrm{Sr}, \mathrm{La})_{2} \mathrm{CuO}_{6+\delta}$ by Resonant Inelastic X-Ray Scattering, Phys. Rev. B 94, 184511 (2016).

[39] X. T. Li, Y. Ding, C. C. He, W. Ruan, P. Cai, C. Ye, Z. Q. Hao, L. Zhao, X. J. Zhou, Q. H. Wang, and Y. Y. Wang, Quasiparticle Interference and Charge Order in a Heavily Overdoped Non-superconducting Cuprate, New J. Phys. 20, 063041 (2018).

[40] J. W. Alldredge, J. Lee, K. McElroy, M. Wang, K. Fujita, Y. Kohsaka, C. Taylor, H. Eisaki, S. Uchida, P. J. Hirschfeld, and J.C. Davis, Evolution of the Electronic Excitation Spectrum with Strongly Diminishing Hole Density in Superconducting $\mathrm{Bi}_{2} \mathrm{Sr}_{2} \mathrm{CaCu}_{2} \mathrm{O}_{8+\delta}$, Nat. Phys. 4, 319 (2008).

[41] I. Zeljkovic, E. J. Main, T. L. Williams, M. C. Boyer, K. Chatterjee, W. D. Wise, Y. Yin, M. Zech, A. Pivonka, T. Kondo, T. Takeuchi, H. Ikuta, J. Wen, Z. Xu, G. D. Gu, E.W. Hudson, and J.E. Hoffman, Scanning Tunnelling Microscopy Imaging of Symmetry-Breaking Structural Distortion in the Bismuth-Based Cuprate Superconductors, Nat. Mater. 11, 585 (2012).

[42] K. Fujita, C. K. Kim, I. Lee, J. Lee, M. H. Hamidian, I. A. Firmo, S. Mukhopadhyay, H. Eisaki, S. Uchida, M. J. Lawler, E. A. Kim, and J.C. Davis, Simultaneous Transitions in Cuprate Momentum-Space Topology and Electronic Symmetry Breaking, Science 344, 612 (2014).

[43] M. H. Hamidian, S. D. Edkins, C. K. Kim, J. C. Davis, A. P. Mackenzie, H. Eisaki, S. Uchida, M. J. Lawler, E. A. Kim, S. Sachdev, and K. Fujita, Atomic-Scale Electronic Structure of the Cuprate d-Symmetry Form Factor Density Wave State, Nat. Phys. 12, 150 (2016).

[44] A. Mesaros, K. Fujita, S. D. Edkins, M. H. Hamidian, H. Eisaki, S. I. Uchida, J. C. S. Davis, M. J. Lawler, and E. A. Kim, Commensurate $4 \mathrm{a}_{0}$-Period Charge Density Modulations throughout the $\mathrm{Bi}_{2} \mathrm{Sr}_{2} \mathrm{CaCu}_{2} \mathrm{O}_{8+x}$ Pseudogap Regime, Proc. Natl. Acad. Sci. U.S.A. 113, 12661 (2016).

[45] P. Choubey, W. L. Tu, T. K. Lee, and P. J. Hirschfeld, Incommensurate Charge Ordered States in the $\mathrm{t}-\mathrm{t}^{\prime}-J$ Model, New J. Phys. 19, 013028 (2017).

[46] S. D. Edkins, A. Kostin, K. Fujita, A. P. Mackenzie, H. Eisaki, S. Uchida, S. Sachdev, M. J. Lawler, E. A. Kim, J.C.S. Davis, and M.H. Hamidian, Magnetic FieldInduced Pair Density Wave State in the Cuprate Vortex Halo, Science 364, 976 (2019).
[47] D. L. Feng, D. H. Lu, K. M. Shen, C. Kim, H. Eisaki, A. Damascelli, R. Yoshizaki, J. Shimoyama, K. Kishio, G. D. Gu, S. Oh, A. Andrus, J. O'Donnell, J. N. Eckstein, and Z. X. Shen, Signature of Superfluid Density in the SingleParticle Excitation Spectrum of $\mathrm{Bi}_{2} \mathrm{Sr}_{2} \mathrm{CaCu}_{2} \mathrm{O}_{8+\delta}$, Science 289, 277 (2000).

[48] Y. Kohsaka, C. Taylor, P. Wahl, A. Schmidt, J. Lee, K. Fujita, J. W. Alldredge, K. McElroy, J. Lee, H. Eisaki, S. Uchida, D. H. Lee, and J.C. Davis, How Cooper Pairs Vanish Approaching the Mott Insulator in $\mathrm{Bi}_{2} \mathrm{Sr}_{2} \mathrm{CaCu}_{2} \mathrm{O}_{8+\delta}$, Nature (London) 454, 1072 (2008).

[49] S. H. Pan, E. W. Hudson, K. M. Lang, H. Eisaki, S. Uchida, and J.C. Davis, Imaging the Effects of Individual Zinc Impurity Atoms on Superconductivity in $\mathrm{Bi}_{2} \mathrm{Sr}_{2} \mathrm{CaCu}_{2} \mathrm{O}_{8+\delta}$, Nature (London) 403, 746 (2000).

[50] S. H. Pan, E. W. Hudson, A. K. Gupta, K. W. Ng, H. Eisaki, S. Uchida, and J. C. Davis, STM Studies of the Electronic Structure of Vortex Cores in $\mathrm{Bi}_{2} \mathrm{Sr}_{2} \mathrm{CaCu}_{2} \mathrm{O}_{8+\delta}$, Phys. Rev. Lett. 85, 1536 (2000).

[51] Y. He, Y. Yin, M. Zech, A. Soumyanarayanan, M. M. Yee, T. Williams, M. C. Boyer, K. Chatterjee, W. D. Wise, I. Zeljkovic, T. Kondo, T. Takeuchi, H. Ikuta, P. Mistark, R. S. Markiewicz, A. Bansil, S. Sachdev, E. W. Hudson, and J. E. Hoffman, Fermi Surface and Pseudogap Evolution in a Cuprate Superconductor, Science 344, 608 (2014).

[52] K. K. Gomes, A. N. Pasupathy, A. Pushp, S. Ono, Y. Ando, and A. Yazdani, Visualizing Pair Formation on the Atomic Scale in the High- $T_{c}$ Superconductor $\mathrm{Bi}_{2} \mathrm{Sr}_{2} \mathrm{CaCu}_{2} \mathrm{O}_{8+\delta}$, Nature (London) 447, 569 (2007).

[53] D. Cho, K. M. Bastiaans, D. Chatzopoulos, G. D. Gu, and M. P. Allan, A Strongly Inhomogeneous Superfluid in an Iron-Based Superconductor, Nature (London) 571, 541 (2019).

[54] J. A. Robertson, S. A. Kivelson, E. Fradkin, A. C. Fang, and A. Kapitulnik, Distinguishing Patterns of Charge Order: Stripes or Checkerboards, Phys. Rev. B 74, 134507 (2006).

[55] E. Fradkin, S. A. Kivelson, M. J. Lawler, J. P. Eisenstein, and A. P. Mackenzie, Nematic Fermi Fluids in Condensed Matter Physics, Annu. Rev. Condens. Matter Phys. 1, 153 (2010).

[56] T. Andrade, A. Krikun, K. Schalm, and J. Zaanen, Doping the Holographic Mott Insulator, Nat. Phys. 14, 1049 (2018). 